\title{
A characterization of missingness at random in a generalized shared-parameter joint modeling framework for longitudinal and time-to-event data, and sensitivity analysis
}

\author{
Edmund Njeru Njagi ${ }^{1}$, Geert Molenberghs' ${ }^{* 1,2}$, Michael G. Kenward ${ }^{3}$, Geert Verbeke ${ }^{2,1}$, \\ and Dimitris Rizopoulos ${ }^{4}$ \\ 1 I-BioStat, Universiteit Hasselt, B-3590 Diepenbeek, Belgium \\ 2 I-BioStat, Katholieke Universiteit Leuven, B-3000 Leuven, Belgium \\ ${ }^{3}$ Department of Medical Statistics, London School of Hygiene and Tropical Medicine, London \\ WC1E7HT, United Kingdom \\ ${ }^{4}$ Department of Biostatistics, Erasmus University Medical Center, NL-3000 CA Rotterdam, The \\ Netherlands
}

Received 10 February 2013; revised 27 March 2014; accepted 18 April 2014

\begin{abstract}
We consider a conceptual correspondence between the missing data setting, and joint modeling of longitudinal and time-to-event outcomes. Based on this, we formulate an extended shared random effects joint model. Based on this, we provide a characterization of missing at random, which is in line with that in the missing data setting. The ideas are illustrated using data from a study on liver cirrhosis, contrasting the new framework with conventional joint models.
\end{abstract}

Keywords: Censoring; Coarsening; Missing at Random; Missingness; Missing not at Random; Pattern-mixture model; Selection model; Shared-parameter model.

Additional supporting information may be found in the online version of this article at the publisher's web-site

\section{Introduction}

In the missing data setting, three main classes of models have been developed: the so-called selection (SEM), pattern-mixture (PMM), and shared-parameter (SPM) frameworks; see for example Molenberghs and Kenward (2007). The SEM and PMM approaches are defined by different factorizations of the joint distribution of the data and the missing value processes. In the SPM, on the other hand, both data and the missing value process are assumed to depend on latent variables, conditional upon which independence is assumed.

Originally due to Rubin (1976), a classification of missing value processes within the SEM has been developed. In a frequentist paradigm, with outcomes only missing, this classification can be expressed as follows: Missing completely at random (MCAR), implying that, conditional upon covariates, the missing value mechanism does not depend on outcomes; missing at random (MAR), implying that conditional on covariates and observed outcomes, the mechanism does not further depend on missing outcomes; and, finally, when MCAR and MAR do not hold, we have a missing not at random (MNAR) process, in which, conditional on covariates and observed outcomes, the missing value mechanism does

*Corresponding author: e-mail: geert.molenberghs@uhasselt.be 
E. N. Njagi et al.: A characterization of missingness at random

depend on unobserved outcomes. The taxonomy has also been transposed to the PMM (Molenberghs et al., 1998) and SPM (Creemers et al., 2011) frameworks.

Given that models for missing data often make unverifiable assumptions about the missing value mechanism, a recurring theme is that of sensitivity analysis (Verbeke and Molenberghs, 2000; Molenberghs and Verbeke, 2005). As assumptions regarding the missing value mechanism are varied, the stability of inferences, or lack thereof, provides a guide on the caution with which the inferences need to be embraced. Though sensitivity analysis has primarily been done under the SEM and PMM frameworks, Creemers et al. (2010) considered it in the context the SPM.

The joint longitudinal and time-to-event setting is slightly different, given that a time-to-event is also collected. An example is in prostate cancer, where, after treatment for cancer, prostate-specific antigen measurements are collected over time, alongside the time-to-disease-recurrence (Law et al., 2002; Yu et al., 2004, 2008). In HIV/AIDS studies, apart from the time to onset of AIDS or death, viral load, and CD4 cell count may be recorded repeatedly over time (DeGruttola and Tu, 1994; Rizopoulos, 2011). The objectives may be threefold: first, to study the survival outcome, accounting for the longitudinal covariate; second, to study the longitudinal outcome, accounting for possibly nonrandom drop-out caused by the occurrence of events; and third, to examine the association structure between the two outcomes (Tsiatis and Davidian, 2004; Rizopoulos et al., 2009; Verbeke et al., 2010; Rizopoulos, 2012).

Among the three objectives, the first one is arguably the most common. This objective is usually achieved within the SPM framework. A submodel for the time-to-event is linked to one for the longitudinal process using a shared latent structure, say a normal random effect, conditional on which independence is assumed (Tsiatis and Davidian, 2004; Verbeke et al., 2010; Rizopoulos, 2011, 2012). Like in the missing data setting, there are challenges. The longitudinal covariate may be measured with error, its values are likewise only available at the specific time points at that the patient appears at the clinic for longitudinal measurements, and the time-to-event may also be censored (Tsiatis and Davidian, 2004).

As such, though interest is on the time-to-event accounting for the "true" longitudinal process, the joint density incorporates not only the censoring but also the visiting and measurement probabilities (Tsiatis and Davidian, 2004). The visiting probabilities represent the process that generates the time points at which measurements are available (Rizopoulos, 2012). To identify the relationship of interest, under likelihood inference, it is assumed that the probabilities of censoring and visiting can depend on past visit times and longitudinal measurements, but not further on the future longitudinal measurements and event time (Tsiatis and Davidian, 2004; Verbeke et al., 2010; Rizopoulos, 2012). These conditions mirror the MAR assumption mentioned earlier. The assumptions are again unverifiable based on the data, raising sensitivity issues. Rizopoulos (2012) for instance considers the second objective mentioned above, and highlights the consideration of different parameterizations of the longitudinal process in the survival submodel, as a possible route for sensitivity analysis.

Undeniably, there is a strong connection between the missing data and the joint longitudinal and time-to-event settings. In this paper, we take a slightly different perspective on joint models than is prevalent in the literature, and argue that conceptually, the two settings actually correspond. Based on this, we build an extended shared random effects joint model, similar in spirit to that of Creemers et al. (2011) in the context of longitudinal data subject to missing observations, but now transposed to the current more complex setting. The added layer of complexity stems from the fact that data can now be coarsened in various ways: the longitudinal sequence can be incomplete; the time-to-event outcome can be censored; both of these can occur simultaneously. Coarsening refers to the phenomenon that data observed are less refined than the, possibly counterfactual, full data.

Within the extended framework, we provide a characterization of MAR, consistent to the one in the missing data setting, and juxtapose it with more conventional joint models. This opens routes for sensitivity analysis.

The organization of the paper is as follows. In the following section, we briefly review missing data concepts, the various modeling frameworks, and the characterization of MAR in each of these frameworks. We review the generalized shared-parameter modeling (GSPM) framework of Creemers 
et al. (2011), and its MAR characterization. In Section 3, we introduce the problem of joint modeling of longitudinal and time-to-event data and illustrate the correspondence between this problem and that of missing data. Based on this, we introduce our extended framework, and derive its MAR characterization. We provide an illustrative application in Section 4, and finally provide some closing remarks in Section 5.

\section{Background on missing data}

\subsection{Notation}

Let $Y_{i j}$ denote the outcome for the $i^{t h}$ subject measured at the $j^{t h}$ occasion, $i=1, \ldots, N, j=1, \ldots, n_{i}$. Additionally, define a missingness indicator, $R_{i j}$, which takes the value 1 if $Y_{i j}$ is observed, and 0 otherwise. We then have $\boldsymbol{Y}_{i}$ and $\boldsymbol{R}_{i}$, representing the measurement and missingness process vectors for subject $i$, respectively. Further partition $\boldsymbol{Y}_{i}$ into its observed and unobserved components $\boldsymbol{Y}_{i}^{o}$ and $\boldsymbol{Y}_{i}^{m}$, respectively. We also define $\boldsymbol{\theta}$ and $\boldsymbol{\psi}$ to be the parameter vectors for the measurement and missingness processes, respectively. We suppress the covariate vector $\boldsymbol{x}_{i}$ in the notation. Hence, we write $f\left(\boldsymbol{y}_{i}, \boldsymbol{r}_{i} \mid \boldsymbol{\theta}, \boldsymbol{\psi}\right)$ for the full data density.

\subsection{Modeling frameworks}

The selection model (SEM) starts from the factorization $f\left(\boldsymbol{y}_{i}, \boldsymbol{r}_{i} \mid \boldsymbol{\theta}, \boldsymbol{\psi}\right)=f\left(\boldsymbol{y}_{i} \mid \boldsymbol{\theta}\right) f\left(\boldsymbol{r}_{i} \mid \boldsymbol{y}_{i}, \boldsymbol{\psi}\right)$, in contrast to the PMM representation that is based upon $f\left(\boldsymbol{y}_{i}, \boldsymbol{r}_{i} \mid \boldsymbol{\theta}, \boldsymbol{\psi}\right)=f\left(\boldsymbol{y}_{i} \mid \boldsymbol{r}_{i}, \boldsymbol{\theta}\right) f\left(\boldsymbol{r}_{i} \mid \boldsymbol{\psi}\right)$. The conventional SPM incorporates a vector of shared latent variables $\boldsymbol{b}_{i}$, conditional upon which independence of the measurement and missingness processes is assumed, that is

$$
f\left(\boldsymbol{y}_{i}, \boldsymbol{r}_{i} \mid \boldsymbol{\theta}, \boldsymbol{\psi}\right)=\int f\left(\boldsymbol{y}_{i} \mid \boldsymbol{b}_{i}, \boldsymbol{\theta}\right) f\left(\boldsymbol{r}_{i} \mid \boldsymbol{b}_{i}, \boldsymbol{\psi}\right) f\left(\boldsymbol{b}_{i}\right) d \boldsymbol{b}_{i}
$$

\subsection{Characterization of missing at random}

First, we consider MAR in the various frameworks. By definition, under the SEM framework, missingness is MAR if $f\left(\boldsymbol{r}_{i} \mid \boldsymbol{y}_{i}, \boldsymbol{\psi}\right)=f\left(\boldsymbol{r}_{i} \mid \boldsymbol{y}_{i}^{o}, \boldsymbol{\psi}\right)$. Under the PMM framework, the missing-data mechanism is MAR if

$$
f\left(\boldsymbol{y}_{i}^{m} \mid \boldsymbol{y}_{i}^{o}, \boldsymbol{r}_{i}, \boldsymbol{\theta}\right)=f\left(\boldsymbol{y}_{i}^{m} \mid \boldsymbol{y}_{i}^{o}, \boldsymbol{\theta}\right)
$$

(Molenberghs et al., 1998). In (2), the so-called predictive distribution of the unobserved outcomes, given the observed ones, is made explicit. Thus, in the PMM setting, MAR can be seen to mean that the unobserved outcomes can be predicted from the observed outcomes and covariates, without further reference to the missingness mechanism.

Under conventional SPM in (1), MAR cannot hold without reducing to MCAR, in which case $\boldsymbol{b}_{i}$ drops from at least one of the factors in the integrand of (1). Creemers et al. (2011) generalized the SPM (GSPM) by expanding the random-effects structure:

$$
\begin{aligned}
& f\left(\boldsymbol{y}_{i}, \boldsymbol{r}_{i} \mid \boldsymbol{g}_{i}, \boldsymbol{h}_{i}, \boldsymbol{j}_{i}, \boldsymbol{k}_{i}, \boldsymbol{\ell}_{i}, \boldsymbol{m}_{i}, \boldsymbol{q}_{i}\right) \\
& \quad=f\left(\boldsymbol{y}_{i}^{o} \mid \boldsymbol{g}_{i}, \boldsymbol{h}_{i}, \boldsymbol{j}_{i}, \boldsymbol{\ell}_{i}\right) f\left(\boldsymbol{y}_{i}^{m} \mid \boldsymbol{y}_{i}^{o}, \boldsymbol{g}_{i}, \boldsymbol{h}_{i}, \boldsymbol{k}_{i}, \boldsymbol{m}_{i}\right) f\left(\boldsymbol{r}_{i} \mid \boldsymbol{g}_{i}, \boldsymbol{j}_{i}, \boldsymbol{k}_{i}, \boldsymbol{q}_{i}\right)
\end{aligned}
$$

The random-effects structure is more general; the random effects $\boldsymbol{g}_{i}$ are shared among all processes, $\boldsymbol{h}_{i}, \boldsymbol{j}_{i}$, and $\boldsymbol{k}_{i}$ are shared between two processes only, while $\boldsymbol{\ell}_{i}, \boldsymbol{m}_{i}$, and $\boldsymbol{q}_{i}$ are specific to one. Using this 
general formulation, the above-mentioned authors established that GSPM (3) is MAR if and only if

$$
\begin{array}{r}
\frac{\int f\left(\boldsymbol{y}_{i}^{o} \mid \boldsymbol{g}_{i}, \boldsymbol{h}_{i}, \boldsymbol{j}_{i}\right) f\left(\boldsymbol{y}_{i}^{m} \mid \boldsymbol{y}_{i}^{o}, \boldsymbol{g}_{i}, \boldsymbol{h}_{i}, \boldsymbol{k}_{i}\right) f\left(\boldsymbol{r}_{i} \mid \boldsymbol{g}_{i}, \boldsymbol{j}_{i}, \boldsymbol{k}_{i}\right) f\left(\boldsymbol{b}_{i}\right) d \boldsymbol{b}_{i}}{\int f\left(\boldsymbol{y}_{i}^{o} \mid \boldsymbol{g}_{i}, \boldsymbol{j}_{i}\right) f\left(\boldsymbol{r}_{i} \mid \boldsymbol{g}_{i}, \boldsymbol{j}_{i}\right) f\left(\boldsymbol{b}_{i}\right) d \boldsymbol{b}_{i}} \\
=\frac{\int f\left(\boldsymbol{y}_{i}^{o} \mid \boldsymbol{g}_{i}, \boldsymbol{h}_{i}\right) f\left(\boldsymbol{y}_{i}^{m} \mid \boldsymbol{y}_{i}^{o}, \boldsymbol{g}_{i}, \boldsymbol{h}_{i}\right) f\left(\boldsymbol{b}_{i}\right) d \boldsymbol{b}_{i}}{f\left(\boldsymbol{y}_{i}^{o}\right)} .
\end{array}
$$

A convenient proper subclass of GSPM (3) that satisfies MAR:

$$
\begin{aligned}
f\left(\boldsymbol{y}_{i}, \boldsymbol{r}_{i} \mid \boldsymbol{g}_{i}, \boldsymbol{h}_{i}, \boldsymbol{j}_{i}, \boldsymbol{k}_{i}, \boldsymbol{\ell}_{i}, \boldsymbol{m}_{i}, \boldsymbol{q}_{i}\right) & =f\left(\boldsymbol{y}_{i}, \boldsymbol{r}_{i} \mid \boldsymbol{j}_{i}, \boldsymbol{\ell}_{i}, \boldsymbol{m}_{i}, \boldsymbol{q}_{i}\right) \\
& =f\left(\boldsymbol{y}_{i}^{o} \mid \boldsymbol{j}_{i}, \boldsymbol{\ell}_{i}\right) f\left(\boldsymbol{y}_{i}^{m} \mid \boldsymbol{y}_{i}^{o}, \boldsymbol{m}_{i}\right) f\left(r_{i} \mid \boldsymbol{j}_{i}, \boldsymbol{q}_{i}\right) .
\end{aligned}
$$

\section{Joint modeling of longitudinal and time-to-event data}

We consider now the setting in which it is intended to observe both a longitudinal and time-to-event outcome between the start and the planned end of a study. We examine three scenarios to illustrate the correspondence between this setting and that of missing data.

Scenario 1. For subjects who drop out before the planned end of the study, we observe longitudinal information prior to drop-out, as well as the censoring time. Consequently, for these subjects, we observe neither the latter part of the longitudinal sequence nor the survival time.

Scenario 2. For subjects who experience the event within the study period, such that the event censors the longitudinal sequence, we observe longitudinal information prior to the event, as well as the survival time. For these subjects, longitudinal data after the event, as well as the censoring time, are unobserved. The latter means, of course, that no censoring occurs.

Scenario 3. Finally, for subjects who reach the end of the study without experiencing the event, full longitudinal information as well as the censoring time are observed. For these subjects, the survival time remains unobserved.

From these three scenarios, we note that this setting always entails a part of the data being observed, and a part unobserved. We must also consider the mechanism that causes the coarsening, which consists of the union of the missingness mechanism in the longitudinal outcome, and a certain choice mechanism, related to the time-to-event outcome, which determines whether either the event time or censoring time is observed. Note that further scenarios are possible, even though we will restrict attention to these three in the current paper. For example, it is possible for the longitudinal process to terminate early, while the patient is still followed for the event.

\subsection{Additional notation}

We extend the notation of Section 2.1 to let $T_{i}$ and $C_{i}$ denote the survival and censoring times, respectively. Additionally, let $D_{i}^{o}=\min \left(T_{i}, C_{i}\right)$, and $D_{i}^{m}=\max \left(T_{i}, C_{i}\right)$.

We also introduce a vector of missingness indicators, $\boldsymbol{R}_{i}^{*}=\left(\boldsymbol{R}_{i}^{\prime}, W_{i}\right)^{\prime}$, where $W_{i}=1$ if the survival time is observed and zero otherwise. The full set of stochastic components is then:

$$
\boldsymbol{Q}_{i}=\left(\boldsymbol{Y}_{i}^{o^{\prime}}, \boldsymbol{Y}_{i}^{m^{\prime}}, D_{i}^{o}, D_{i}^{m}, \boldsymbol{R}_{i}^{*^{\prime}}\right)^{\prime}=\left(\boldsymbol{Z}_{i}^{o \prime}, \boldsymbol{Z}_{i}^{m^{\prime}}, \boldsymbol{R}_{i}^{*}\right)^{\prime},
$$

where $\boldsymbol{Z}_{i}^{o}=\left(\boldsymbol{Y}_{i}^{o^{\prime}}, D_{i}^{o}\right)^{\prime}$ and $\boldsymbol{Z}_{i}^{m}=\left(\boldsymbol{Y}_{i}^{m^{\prime}}, D_{i}^{m}\right)^{\prime}$. Hence, we can represent the information in a form that parallels that for incomplete longitudinal data, but with each of the three vectors combining both longitudinal and time-to-event information. The information in $\boldsymbol{Z}_{i}^{o}$ and $\boldsymbol{Z}_{i}^{m}$ depends on the particular scenario, which sets this notation apart from that found in the literature. 


\subsection{The extended framework}

Define the following shared random-effects model for these data:

$$
f\left(\boldsymbol{y}_{i}^{o}, \boldsymbol{y}_{i}^{m}, d_{i}^{o}, d_{i}^{m}, \boldsymbol{r}_{i}^{*} \mid \boldsymbol{b}_{i}\right)=f\left(\boldsymbol{y}_{i}^{o} \mid \boldsymbol{b}_{i}\right) f\left(\boldsymbol{y}_{i}^{m} \mid \boldsymbol{y}_{i}^{o}, \boldsymbol{b}_{i}\right) f\left(d_{i}^{o} \mid \boldsymbol{b}_{i}\right) f\left(d_{i}^{m} \mid d_{i}^{o}, \boldsymbol{b}_{i}\right) f\left(\boldsymbol{r}_{i}{ }^{*} \mid \boldsymbol{b}_{i}\right) .
$$

Here, $\boldsymbol{b}_{i}$ encompasses an elaborate random effects structure, which contains the following 31 sets of random effects: 1 shared between all five components, five shared between four components, 10 shared between three components, another 10 shared between two components, and five specific to a single component. These random effects are assumed independent. It is unwieldy to spell them all out as was done in (3), but the nature of the decomposition is identical. As we see below, the advantage of such a framework is that appropriate subsets of random effects can be chosen so that MAR holds.

We make the following points. First, model (7) is the generic shared random-effects model for this setting under this factorization. Such a general structure implies, for instance, that at the time of drop-out, there are processes that may stop, while other processes may get modified. Second, specific submodels may be considered that are deemed appropriate for the application at hand. This is important, because the full set of random effects will typically be too elaborate for practical purposes. Third, for every application, it is important to consider the implication of the corresponding simplification, especially in terms of the underlying coarsening mechanism. It is then important to carefully distinguish between the case where the coarsening does not depend on unobserved measurements, on the one hand, and the coarsening mechanism is MAR, on the other hand. Fourth, the extended model in (7) is obviously based on conditional independence assumptions: given the collection of random effects $\boldsymbol{b}_{i}$, the processes $\boldsymbol{y}_{i}, \boldsymbol{d}_{i}$, and $\boldsymbol{r}_{i}$ are independent of one another. Of course, if all 31 random effects would be present, there still would be a rich association structure present between the various outcomes, which may be simplified by omitting one or more of these components, as will be done to allow for MAR in the next section. Fifth, the model a priori allows for dependence between $d_{i}^{o}$ and $d_{i}^{m}$, regardless of the random-effects structure adopted, stemming from the penultimate factor on the right hand side of (7). In other words, $T_{i}$ and $C_{i}$ would be allowed to depend on one another. In full generality, this would be problematic because Tsiatis (1975) has shown that there is no information available from the data on the joint distribution of $\left(T_{i}, C_{i}\right)$. The simplest way out is to force independence, by writing the corresponding factor as $f\left(d_{i}^{m} \mid d_{i}^{o}, \boldsymbol{b}_{i}\right)=f\left(d_{i}^{m} \mid \boldsymbol{b}_{i}\right)$, with perhaps even the random effect removed. However, it is not our purpose to provide a general framework of which every member is identifiable, but rather one that generates more identifiable special cases than conventionally considered, in particular the MAR version of the next section. In addition, classes of nonidentifiable models can be considered as part of a sensitivity analysis.

\subsection{Characterization of missing at random}

Extended model (7) allows for a characterization of MAR, in the same spirit as (4). We can define MAR by either starting from a SEM-based or from a PMM-based factorization of the model. Under a SEM factorization, the requirement is:

$$
f\left(\boldsymbol{r}_{i}^{*} \mid \boldsymbol{y}_{i}^{o}, \boldsymbol{y}_{i}^{m}, d_{i}^{o}, d_{i}^{m}\right)=f\left(\boldsymbol{r}_{i}^{*} \mid \boldsymbol{y}_{i}^{o}, d_{i}^{o}\right)
$$

implying

$$
\frac{f\left(\boldsymbol{y}_{i}^{o}, \boldsymbol{y}_{i}^{m}, d_{i}^{o}, d_{i}^{m}, \boldsymbol{r}_{i}^{*}\right)}{f\left(\boldsymbol{y}_{i}^{o}, \boldsymbol{y}_{i}^{m}, d_{i}^{o}, d_{i}^{m}\right)}=\frac{f\left(\boldsymbol{y}_{i}^{o}, d_{i}^{o}, \boldsymbol{r}_{i}^{*}\right)}{f\left(\boldsymbol{y}_{i}^{o}, d_{i}^{o}\right)} .
$$

From a PMM perspective, the requirement is:

$$
\frac{f\left(\boldsymbol{y}_{i}^{o}, \boldsymbol{y}_{i}^{m}, d_{i}^{o}, d_{i}^{m}, \boldsymbol{r}_{i}^{*}\right)}{f\left(\boldsymbol{y}_{i}^{o}, d_{i}^{o}, \boldsymbol{r}_{i}^{*}\right)}=\frac{f\left(\boldsymbol{y}_{i}^{o}, \boldsymbol{y}_{i}^{m}, d_{i}^{o}, d_{i}^{m}\right)}{f\left(\boldsymbol{y}_{i}^{o}, d_{i}^{o}\right)} .
$$


Using the specific form of (7), MAR holds if and only if:

$$
\begin{aligned}
& \frac{\int f\left(\boldsymbol{y}_{i}^{o} \mid \boldsymbol{b}_{i}\right) f\left(\boldsymbol{y}_{i}^{m} \mid \boldsymbol{y}_{i}^{o}, \boldsymbol{b}_{i}\right) f\left(d_{i}^{o} \mid \boldsymbol{b}_{i}\right) f\left(d_{i}^{m} \mid d_{i}^{o}, \boldsymbol{b}_{i}\right) f\left(\boldsymbol{r}_{i}^{*} \mid \boldsymbol{b}_{i}\right) f\left(\boldsymbol{b}_{i}\right) d \boldsymbol{b}_{i}}{\int f\left(\boldsymbol{y}_{i}^{o} \mid \boldsymbol{b}_{i}\right) f\left(d_{i}^{o} \mid \boldsymbol{b}_{i}\right) f\left(\boldsymbol{r}_{i}^{*} \mid \boldsymbol{b}_{i}\right) f\left(\boldsymbol{b}_{i}\right) d \boldsymbol{b}_{i}} \\
& =\frac{\int f\left(\boldsymbol{y}_{i}^{o} \mid \boldsymbol{b}_{i}\right) f\left(\boldsymbol{y}_{i}^{m} \mid \boldsymbol{y}_{i}^{o}, \boldsymbol{b}_{i}\right) f\left(d_{i}^{o} \mid \boldsymbol{b}_{i}\right) f\left(d_{i}^{m} \mid d_{i}^{o}, \boldsymbol{b}_{i}\right) f\left(\boldsymbol{b}_{i}\right) d \boldsymbol{b}_{i}}{\int f\left(\boldsymbol{y}_{i}^{o} \mid \boldsymbol{b}_{i}\right) f\left(d_{i}^{o} \mid \boldsymbol{b}_{i}\right) f\left(\boldsymbol{b}_{i}\right) d \boldsymbol{b}_{i}}
\end{aligned}
$$

Recall that $\boldsymbol{b}_{i}$ as used here generically refers to the set of random effects that apply to the factor concerned.

\subsection{A subclass of the generalized SPM}

Consider the following subclass of model (7):

$$
\begin{aligned}
& f\left(\boldsymbol{y}_{i}^{o}, \boldsymbol{y}_{i}^{m}, d_{i}^{o}, d_{i}^{m}, \boldsymbol{r}_{i}^{*} \mid \boldsymbol{b}_{i}\right) \\
& =f\left(\boldsymbol{y}_{i}^{o} \mid \boldsymbol{g}_{i}, \boldsymbol{h}_{i}, \boldsymbol{k}_{i}\right) f\left(\boldsymbol{y}_{i}^{m} \mid \boldsymbol{y}_{i}^{o}, \boldsymbol{m}_{i}\right) f\left(d_{i}^{o} \mid \boldsymbol{g}_{i}, \boldsymbol{h}_{i}, \boldsymbol{\ell}_{i}\right) f\left(d_{i}^{m} \mid d_{i}^{o}, \boldsymbol{m}_{i}\right) f\left(\boldsymbol{r}_{i}^{*} \mid \boldsymbol{g}_{i}, \boldsymbol{k}_{i}, \boldsymbol{\ell}_{i}\right),
\end{aligned}
$$

where $\boldsymbol{g}_{i}, \boldsymbol{h}_{i}, \boldsymbol{k}_{i}, \boldsymbol{\ell}_{i}$, and $\boldsymbol{m}_{i}$ are part of the 31 sets of random effects described earlier. Note that under this subclass, the random effects driving the missing-data components $\boldsymbol{y}_{i}{ }^{m}$ and $d_{i}^{m}$ do not appear in any of the other three stochastic components. Next, we show that this subclass satisfies the MAR property. Let $\widetilde{\boldsymbol{b}}_{i}$ be shorthand for the set of random effects $\left(\boldsymbol{g}_{i}, \boldsymbol{h}_{i}, \boldsymbol{k}_{i}, \boldsymbol{\ell}_{i}\right.$, and $\left.\boldsymbol{m}_{i}\right)$, and $\overline{\boldsymbol{b}}_{i}$ be shorthand for the same set but excluding $\boldsymbol{m}_{i}$. Then, from a SEM-based factorization,

$$
\begin{aligned}
& f\left(\boldsymbol{r}_{i}^{*} \mid \boldsymbol{y}_{i}^{o}, \boldsymbol{y}_{i}^{m}, d_{i}^{o}, d_{i}^{m}\right) \\
& =\frac{f\left(\boldsymbol{y}_{i}^{o}, \boldsymbol{y}_{i}^{m}, d_{i}^{o}, d_{i}^{m}, \boldsymbol{r}_{i}^{*}\right)}{f\left(\boldsymbol{y}_{i}^{o}, \boldsymbol{y}_{i}^{m}, d_{i}^{o}, d_{i}^{m}\right)} \\
& =\frac{\int_{\widetilde{\boldsymbol{b}}_{i}} f\left(\boldsymbol{y}_{i}^{o} \mid \boldsymbol{g}_{i}, \boldsymbol{h}_{i}, \boldsymbol{k}_{i}\right) f\left(\boldsymbol{y}_{i}^{m} \mid \boldsymbol{y}_{i}^{o}, \boldsymbol{m}_{i}\right) f\left(d_{i}^{o} \mid \boldsymbol{g}_{i}, \boldsymbol{h}_{i}, \boldsymbol{\ell}_{i}\right) f\left(d_{i}^{m} \mid d_{i}^{o}, \boldsymbol{m}_{i}\right) f\left(\boldsymbol{r}_{i}^{*} \mid \boldsymbol{g}_{i}, \boldsymbol{k}_{i}, \boldsymbol{\ell}_{i}\right) f\left(\widetilde{\boldsymbol{b}}_{i}\right) d \widetilde{\boldsymbol{b}}_{i}}{\int_{\boldsymbol{r}_{i}^{*}} \int_{\widetilde{\boldsymbol{b}}_{i}} f\left(\boldsymbol{y}_{i}^{o} \mid \boldsymbol{g}_{i}, \boldsymbol{h}_{i}, \boldsymbol{k}_{i}\right) f\left(\boldsymbol{y}_{i}^{m} \mid \boldsymbol{y}_{i}^{o}, \boldsymbol{m}_{i}\right) f\left(d_{i}^{o} \mid \boldsymbol{g}_{i}, \boldsymbol{h}_{i}, \boldsymbol{\ell}_{i}\right) f\left(d_{i}^{m} \mid d_{i}^{o}, \boldsymbol{m}_{i}\right) f\left(\boldsymbol{r}_{i}^{*} \mid \boldsymbol{g}_{i}, \boldsymbol{k}_{i}, \boldsymbol{\ell}_{i}\right) f\left(\widetilde{\boldsymbol{b}}_{i}\right) d \widetilde{\boldsymbol{b}}_{i} d \boldsymbol{r}_{i}^{*}} \\
& =\frac{\int_{\boldsymbol{m}_{i}} f\left(\boldsymbol{y}_{i}^{m} \mid \boldsymbol{y}_{i}^{o}, \boldsymbol{m}_{i}\right) f\left(d_{i}^{m} \mid d_{i}^{o}, \boldsymbol{m}_{i}\right) f\left(\boldsymbol{m}_{i}\right) d \boldsymbol{m}_{i}}{\int_{\boldsymbol{m}_{i}} f\left(\boldsymbol{y}_{i}^{m} \mid \boldsymbol{y}_{i}^{o}, \boldsymbol{m}_{i}\right) f\left(d_{i}^{m} \mid d_{i}^{o}, \boldsymbol{m}_{i}\right) f\left(\boldsymbol{m}_{i}\right) d \boldsymbol{m}_{i}} \\
& \times \frac{\int_{\overline{\boldsymbol{b}}_{i}} f\left(\boldsymbol{y}_{i}^{o} \mid \boldsymbol{g}_{i}, \boldsymbol{h}_{i}, \boldsymbol{k}_{i}\right) f\left(d_{i}^{o} \mid \boldsymbol{g}_{i}, \boldsymbol{h}_{i}, \boldsymbol{\ell}_{i}\right) f\left(\boldsymbol{r}_{i}^{*} \mid \boldsymbol{g}_{i}, \boldsymbol{k}_{i}, \boldsymbol{\ell}_{i}\right) f\left(\overline{\boldsymbol{b}}_{i}\right) d \overline{\boldsymbol{b}}_{i}}{\int_{\boldsymbol{r}_{i}^{*}} \int_{\overline{\boldsymbol{b}}_{i}} f\left(\boldsymbol{y}_{i}^{o} \mid \boldsymbol{g}_{i}, \boldsymbol{h}_{i}, \boldsymbol{k}_{i}\right) f\left(d_{i}^{o} \mid \boldsymbol{g}_{i}, \boldsymbol{h}_{i}, \boldsymbol{\ell}_{i}\right) f\left(\boldsymbol{r}_{i}^{*} \mid \boldsymbol{g}_{i}, \boldsymbol{k}_{i}, \boldsymbol{\ell}_{i}\right) f\left(\overline{\boldsymbol{b}}_{i}\right) d \overline{\boldsymbol{b}}_{i} d \boldsymbol{r}_{i}^{*}} \\
& =\frac{f\left(\boldsymbol{y}_{i}^{o}, d_{i}^{o}, \boldsymbol{r}_{i}^{*}\right)}{f\left(\boldsymbol{y}_{i}^{o}, d_{i}^{o}\right)}=f\left(\boldsymbol{r}_{i}^{*} \mid \boldsymbol{y}_{i}^{o}, d_{i}^{o}\right) .
\end{aligned}
$$

Alternatively, one could start from a PMM-based factorization. In this case,

$$
\begin{aligned}
f\left(\boldsymbol{y}_{i}^{m}, d_{i}^{m} \mid \boldsymbol{y}_{i}^{o}, d_{i}^{o}, \boldsymbol{r}_{i}^{*}\right) \\
\quad=\frac{f\left(\boldsymbol{y}_{i}^{o}, \boldsymbol{y}_{i}^{m}, d_{i}^{o}, d_{i}^{m}, \boldsymbol{r}_{i}^{*}\right)}{f\left(\boldsymbol{y}_{i}^{o}, d_{i}^{o}, \boldsymbol{r}_{i}^{*}\right)}
\end{aligned}
$$




$$
\begin{aligned}
& \int_{\widetilde{\boldsymbol{b}}} f\left(\boldsymbol{y}_{i}^{o} \mid \boldsymbol{g}_{i}, \boldsymbol{h}_{i}, \boldsymbol{k}_{i}\right) f\left(\boldsymbol{y}_{i}^{m} \mid \boldsymbol{y}_{i}^{o}, \boldsymbol{m}_{i}\right) f\left(d_{i}^{o} \mid \boldsymbol{g}_{i}, \boldsymbol{h}_{i}, \boldsymbol{\ell}_{i}\right) f\left(d_{i}^{m} \mid d_{i}^{o}, \boldsymbol{m}_{i}\right) f\left(\boldsymbol{r}_{i}^{*} \mid \boldsymbol{g}_{i}, \boldsymbol{k}_{i}, \boldsymbol{\ell}_{i}\right) f\left(\widetilde{\boldsymbol{b}}_{i}\right) d \widetilde{\boldsymbol{b}}_{i} \\
& =\overline{\int_{d_{i}^{m}} \int_{\boldsymbol{y}_{i}^{m}} \int_{\widetilde{\boldsymbol{b}}_{i}} f\left(\boldsymbol{y}_{i}^{o} \mid \boldsymbol{g}_{i}, \boldsymbol{h}_{i}, \boldsymbol{k}_{i}\right) f\left(\boldsymbol{y}_{i}^{m} \mid \boldsymbol{y}_{i}^{o}, \boldsymbol{m}_{i}\right) f\left(d_{i}^{o} \mid \boldsymbol{g}_{i}, \boldsymbol{h}_{i}, \boldsymbol{\ell}_{i}\right) f\left(d_{i}^{m} \mid d_{i}^{o}, \boldsymbol{m}_{i}\right) f\left(\boldsymbol{r}_{i}^{*} \mid \boldsymbol{g}_{i}, \boldsymbol{k}_{i}, \boldsymbol{\ell}_{i}\right) f\left(\widetilde{\boldsymbol{b}}_{i}\right) d \widetilde{\boldsymbol{b}}_{i} d \boldsymbol{y}_{i}^{m} d d_{i}^{m}} \\
& =\frac{\int_{\boldsymbol{m}_{i}} f\left(\boldsymbol{y}_{i}^{m} \mid \boldsymbol{y}_{i}^{o}, \boldsymbol{m}_{i}\right) f\left(d_{i}^{m} \mid d_{i}^{o}, \boldsymbol{m}_{i}\right) f\left(\boldsymbol{m}_{i}\right) d \boldsymbol{m}_{i}}{\int_{d_{i}^{m}} \int_{\boldsymbol{y}_{i}^{m}} \int_{\boldsymbol{m}_{i}} f\left(\boldsymbol{y}_{i}^{m} \mid \boldsymbol{y}_{i}^{o}, \boldsymbol{m}_{i}\right) f\left(d_{i}^{m} \mid d_{i}^{o}, \boldsymbol{m}_{i}\right) f\left(\boldsymbol{m}_{i}\right) d \boldsymbol{m}_{i} d \boldsymbol{y}_{i}^{m} d d_{i}^{m}} \\
& \times \frac{\int_{\overline{\boldsymbol{b}}} f\left(\boldsymbol{y}_{i}^{o} \mid \boldsymbol{g}_{i}, \boldsymbol{h}_{i}, \boldsymbol{k}_{i}\right) f\left(d_{i}^{o} \mid \boldsymbol{g}_{i}, \boldsymbol{h}_{i}, \boldsymbol{\ell}_{i}\right) f\left(\boldsymbol{r}_{i}^{*} \mid \boldsymbol{g}_{i}, \boldsymbol{k}_{i}, \boldsymbol{\ell}_{i}\right) f\left(\overline{\boldsymbol{b}}_{i}\right) d \overline{\boldsymbol{b}}_{i}}{\int_{\overline{\boldsymbol{b}}_{i}} f\left(\boldsymbol{y}_{i}^{o} \mid \boldsymbol{g}_{i}, \boldsymbol{h}_{i}, \boldsymbol{k}_{i}\right) f\left(d_{i}^{o} \mid \boldsymbol{g}_{i}, \boldsymbol{h}_{i}, \boldsymbol{\ell}_{i}\right) f\left(\boldsymbol{r}_{i}^{*} \mid \boldsymbol{g}_{i}, \boldsymbol{k}_{i}, \boldsymbol{\ell}_{i}\right) f\left(\overline{\boldsymbol{b}}_{i}\right) d \overline{\boldsymbol{b}}_{i}} \\
& =f\left(\boldsymbol{y}_{i}^{m}, d_{i}^{m} \mid \boldsymbol{y}_{i}^{o}, d_{i}^{o}\right) \text {. }
\end{aligned}
$$

The above results show that the subclass satisfies MAR. Therefore, a sufficient condition for our extended model to satisfy MAR is that the random effects influencing the observed measurements and/or the coarsening mechanism do not influence the missing measurements, given the observed ones. This is equivalent to the condition that all information about the missing measurements stems from the observed measurements and covariates only.

\subsection{An MAR counterpart to an extended shared-parameter joint model for longitudinal and time-to-event data}

The developments in Sections 3.3 and 3.4 allow us to construct an MAR counterpart for any member of the extended model (7), with exactly the same fit to the observed data. This can be done by integrating over the distribution of the missing components given the observed ones (Molenberghs et al., 2008). Therefore, $f\left(\boldsymbol{y}_{i}^{m} \mid \boldsymbol{y}_{i}^{o}, \boldsymbol{b}_{i}\right)$ and $f\left(d_{i}^{m} \mid d_{i}^{o}, \boldsymbol{b}_{i}\right)$ in (7) need to be replaced with, respectively,

$$
\begin{aligned}
h\left(\boldsymbol{y}_{i}^{m} \mid \boldsymbol{y}_{i}^{o}, \boldsymbol{m}_{i}^{*}\right) & =\int_{\boldsymbol{b}_{i}^{*}} f\left(\boldsymbol{y}_{i}^{m} \mid \boldsymbol{y}_{i}^{o}, \boldsymbol{b}_{i}\right) d \boldsymbol{b}_{i}^{*}, \\
h\left(d_{i}^{m} \mid d_{i}^{o}, \boldsymbol{m}_{i}^{*}\right) & =\int_{\boldsymbol{b}_{i}^{*}} f\left(d_{i}^{m} \mid d_{i}^{o}, \boldsymbol{b}_{i}\right) d \boldsymbol{b}_{i}^{*},
\end{aligned}
$$

where integration over $\boldsymbol{b}_{i}^{*}$ is over all random effects in the full set $\boldsymbol{b}_{i}$, except possibly those that are specific to either $\boldsymbol{y}_{i}^{m}$, or $d_{i}^{m}$, or both.

This nonuniqueness is an important and somewhat disconcerting point. At first, it may seem that it dismisses the use of the proposed framework. However, it is important to see that this is not particular to the model described here. Rather, it always occurs when data are incomplete, as brought forward in Molenberghs and Verbeke (2004). The same is true for models with unobservables such as random effects, as studied by Verbeke and Molenberghs (2011). More generally, whenever the model specifies more than what is available in the data, such nonuniqueness occurs. This has been brought forward in Molenberghs et al. (2012). It means that great care is needed when interpreting results from models with incomplete and censored observations, just as well as for random-effects models, models with latent variables and latent classes, factor-analytic models, etc. This implies that apart from goodness-of-fit, which still has a place but only addresses how well a model described the observed data, sensitivity analysis needs to be done, studying how assumptions about the unobservables, given the observables, influence the inferences drawn. The advantage of our framework is that this is clearly brought to the foreground, reducing the risk of a false sense of security (Creemers et al., 2010).

Obviously, if for instance $\boldsymbol{b}_{i}$ above is a single common random effect, then the marginalization provided above means that if patients' estimated longitudinal profiles would be extended beyond the 
E. N. Njagi et al.: A characterization of missingness at random

point of censoring, then, for a given combination of baseline covariates, the MAR model would reduce the predicted postcensoring trajectories to a common profile. The MNAR model would produce different trajectories. This is of course because the subject-specific effects (random effects) would come then into play under the MNAR, while the same is not the case under the MAR. In addition, if, for censored patients, we would consider their predicted hazard for death, then, for patients having the same combination of baseline covariates, the MAR would produce a common predicted hazard curve, while the MNAR would produce different curves.

\subsection{A narrow definition, and its limitations}

We will now adopt a narrow definition of a joint model, and examine its main limitation, namely, that it defies an MAR characterization.

Before considering the narrow definition, we first set out some results on MAR in the PMM and SEM settings. We can then use a decomposition either in a PMM format:

$$
f\left(\boldsymbol{z}_{i}^{o}, \boldsymbol{z}_{i}^{m}, \boldsymbol{r}_{i}^{*} \mid \boldsymbol{\theta}^{*}, \boldsymbol{\psi}^{*}\right)=f\left(\boldsymbol{z}_{i}^{o} \mid \boldsymbol{r}_{i}^{*}, \boldsymbol{\theta}^{*}\right) f\left(\boldsymbol{z}_{i}^{m} \mid \boldsymbol{z}_{i}^{o}, \boldsymbol{r}_{i}^{*}, \boldsymbol{\theta}^{*}\right) f\left(\boldsymbol{r}_{i}^{*} \mid \boldsymbol{\psi}^{*}\right),
$$

or in a SEM format:

$$
f\left(\boldsymbol{z}_{i}^{o}, \boldsymbol{z}_{i}^{m}, \boldsymbol{r}_{i}^{*} \mid \boldsymbol{\theta}^{* *}, \boldsymbol{\psi}^{* *}\right)=f\left(\boldsymbol{z}_{i}^{o}, z_{i}^{m} \mid \boldsymbol{\theta}^{* *}\right) f\left(\boldsymbol{r}_{i}^{*} \mid \boldsymbol{z}_{i}^{o}, \boldsymbol{z}_{i}^{m}, \boldsymbol{\psi}^{* *}\right) .
$$

Note that parameters are framework-specific. Further, the number of components in $\boldsymbol{Z}_{i}^{o}$ and $\boldsymbol{Z}_{i}^{m}$ varies from subject to subject, in line with what is common in the missing data literature (Rubin, 1976; Little and Rubin, 2002).

In a PMM, MAR is:

$$
f\left(\boldsymbol{z}_{i}^{m} \mid \boldsymbol{z}_{i}^{o}, \boldsymbol{r}_{i}^{*}, \boldsymbol{\theta}^{*}\right)=f\left(z_{i}^{m} \mid \boldsymbol{z}_{i}^{o}, \boldsymbol{\theta}^{*}\right),
$$

whereas in a SEM it is:

$$
f\left(\boldsymbol{r}_{i}^{*} \mid \boldsymbol{z}_{i}^{o}, \boldsymbol{z}_{i}^{m}, \boldsymbol{\psi}^{* *}\right)=f\left(\boldsymbol{r}_{i}^{*} \mid \boldsymbol{z}_{i}^{o}, \boldsymbol{\psi}^{* *}\right) .
$$

Equation (18) implies that the distribution of the unobserved outcomes given the observed ones does not further depend on the coarsening mechanism.

The narrow definition assumes a single common underlying random-effects structure, as opposed to our extended approach. Decomposing this model in a SEM fashion:

$$
\begin{aligned}
& f\left(\boldsymbol{z}_{i}^{o}, \boldsymbol{z}_{i}^{m}, \boldsymbol{r}_{i}^{*}, \boldsymbol{b}_{i}^{* *} \mid \boldsymbol{\theta}^{* *}, \boldsymbol{\psi}^{* *}, \boldsymbol{\omega}^{*}\right) \\
& \quad=f\left(\boldsymbol{z}_{i}^{o} \mid \boldsymbol{b}_{i}^{* *}, \boldsymbol{\theta}^{* *}\right) f\left(\boldsymbol{z}_{i}^{m} \mid \boldsymbol{z}_{i}^{o}, \boldsymbol{b}_{i}^{* *}, \boldsymbol{\theta}^{* *}\right) f\left(\boldsymbol{r}_{i}^{*} \mid \boldsymbol{z}_{i}^{o}, \boldsymbol{z}_{i}^{m}, \boldsymbol{b}_{i}^{* *}, \boldsymbol{\psi}^{* *}\right) f\left(\boldsymbol{b}_{i}^{* *} \mid \boldsymbol{\omega}^{*}\right), \\
& \quad=f\left(\boldsymbol{z}_{i}^{o} \mid \boldsymbol{b}_{i}^{* *}, \boldsymbol{\theta}^{* *}\right) f\left(\boldsymbol{z}_{i}^{m} \mid \boldsymbol{z}_{i}^{o}, \boldsymbol{b}_{i}^{* *}, \boldsymbol{\theta}^{* *}\right) f\left(\boldsymbol{r}_{i}^{*} \mid \boldsymbol{b}_{i}^{* *}, \boldsymbol{\psi}^{* *}\right) f\left(\boldsymbol{b}_{i}^{* *} \mid \boldsymbol{\omega}^{*}\right),
\end{aligned}
$$

with $\omega^{*}$ parameterizing the random-effects distribution, and the step from (20) to (21) following from conditional independence. Formulation (21) means that coarsening cannot depend on the unobserved longitudinal measurements and the future time (whether survival or censoring), given the random effect. Hence, by construction, we have MNAR, unless in the trivial case where the random effect drops out from either the $Z$-factors, or from the $r$-factor, or from both. In the latter case MCAR applies. As stated in Creemers et al. (2011) for longitudinal data, this means that such a narrow formulation cannot admit MAR. This is in contrast to our results in Sections 3.3 and 3.4, where MAR conditions are established for the extended framework. 


\section{The liver cirrhosis data}

These data are from a randomized clinical trial, conducted in Copenhagen, of which the goal was to assess whether Prednisone prolonged survival in patients with liver cirrhosis (Andersen et al., 1993). Liver cirrhosis is a disease in which the liver function deteriorates due to injury. Patients were enrolled between 1962-1969, randomized to either Prednisone or placebo, and followed-up until 1974. Follow-up visits were planned at 3, 6, and 12 months after treatment, and once a year thereafter. We use data on 251 and 237 patients from the Prednisone and placebo groups, respectively. Among other variables recorded at follow-up were several biochemical variables, including bilirubin, albumin, and prothrombin. In this analysis, we consider the quasi-continuous prothrombin index, viewed as a marker for severity of liver fibrosis.

We formulate an extended model for these data, following the logic of the framework proposed, and illustrate the implications of MAR and MNAR. To this end, we set out by considering the following decomposition into five factors, dropping the subject index $i$ from notation, to avoid clutter:

$$
\underline{f\left(\boldsymbol{y}^{o} \mid \boldsymbol{g}, \boldsymbol{h}, \boldsymbol{k}\right)} f\left(\boldsymbol{y}^{m} \mid \boldsymbol{y}^{o}, \boldsymbol{m}\right) \underline{f\left(d^{o} \mid \boldsymbol{g}, \boldsymbol{h}, \ell\right)} f\left(d^{m} \mid d^{o}, \boldsymbol{m}\right) \underline{f(\boldsymbol{r} \mid \boldsymbol{g}, \boldsymbol{k}, \ell)} .
$$

The three underlined factors are identifiable from data, whereas the other two are not. Note that (22) is consistent with MAR, because there is no random-effects link between the unidentifiable factors and the identifiable ones.

We need to distinguish between the situations where either an event or censoring occurs. First, in both cases, the factors

$$
\begin{aligned}
& f\left(\boldsymbol{y}^{o} \mid \boldsymbol{g}, \boldsymbol{h}, \boldsymbol{k}\right), \\
& f(\boldsymbol{r} \mid \boldsymbol{g}, \boldsymbol{k}, \boldsymbol{\ell})
\end{aligned}
$$

are identifiable, with the factor

$$
f\left(\boldsymbol{y}^{m} \mid \boldsymbol{y}^{o}, \boldsymbol{m}\right)
$$

unidentifiable. Second, in case an event occurs, the additional identifiable factor is:

$$
f\left(d^{o}=t \mid \boldsymbol{g}, \boldsymbol{h}, \boldsymbol{\ell}\right)
$$

with the final unidentifiable factor equal to:

$$
f\left(d^{m}=c \mid d^{o}=t, \boldsymbol{m}\right) .
$$

Third, when censoring occurs, the additional identifiable factor is:

$$
f(c, T \geq C \mid \boldsymbol{g}, \boldsymbol{h}, \boldsymbol{\ell})
$$

and the remaining unidentifiable one takes the form:

$$
f\left(d^{m}=t \mid c, T \geq C, \boldsymbol{m}\right) .
$$

In both cases, data-based modeling of the identifiable factors, combined with any choice for the unidentifiable ones, leads to an MAR model. Deviations from MNAR can be studied by inserting additional random effects, present in the identifiable factors, into the unidentifiable components.

Most factors are straightforward to model. Factor (28) is evaluated as follows:

$$
f(c, T \geq C \mid \boldsymbol{g}, \boldsymbol{h}, \boldsymbol{\ell})=\int_{t=c}^{t=\infty} f(c, t \mid \boldsymbol{g}, \boldsymbol{h}, \boldsymbol{\ell}) d t
$$




$$
\begin{aligned}
& =f(c \mid \boldsymbol{g}, \boldsymbol{h}, \boldsymbol{\ell}) \int_{t=c}^{t=\infty} f(t \mid c, \boldsymbol{g}, \boldsymbol{h}, \boldsymbol{\ell}) d t \\
& =f(c \mid \boldsymbol{g}, \boldsymbol{h}, \boldsymbol{\ell})\{1-F(t=c \mid c, \boldsymbol{g}, \boldsymbol{h}, \boldsymbol{\ell})\} .
\end{aligned}
$$

Turning to the liver cirrhosis data, we opt for a single random effect, $\boldsymbol{g}$; that is the random effects $\boldsymbol{h}, \boldsymbol{k}$, and $\boldsymbol{\ell}$ in (22) are dropped. For the longitudinally recorded prothrombin index, we assume the following linear-mixed model:

$$
Y_{i j}^{o}=\beta_{0}+\beta_{1} v_{i j}+\beta_{2} X_{i}+\beta_{3} X_{i} v_{i j}+g_{i}+\varepsilon_{i j},
$$

where $g_{i} \sim N\left(0, \sigma_{g}^{2}\right), \varepsilon_{i j} \sim N\left(0, \sigma^{2}\right)$, and $g_{i}$ and $\varepsilon_{i j}$ are independent. Further, $v_{i j}$ is the time at which the $j^{\text {th }}$ measurement for the $i$ th individual was taken, and $X_{i}$ the treatment indicator. While the main effect of treatment is expected to be zero, stemming from randomization, it is kept in the model to absorb small departures in randomization equilibrium. Model (31) completes the specification for (23).

For the survival time $T$ and censoring time $C$, we assume separate proportional hazards models, with exponential baseline:

$$
\begin{aligned}
& f_{i}\left(t \mid g_{i}\right)=\lambda_{1} \exp \left(X_{i} \xi_{1}+\kappa_{1} g_{i}\right) \exp \left\{-\lambda_{1} t \exp \left(X_{i} \xi_{1}+\kappa_{1} g_{i}\right)\right\}, \\
& f_{i}\left(c \mid g_{i}\right)=\lambda_{2} \exp \left(X_{i} \xi_{2}+\kappa_{2} g_{i}\right) \exp \left\{-\lambda_{2} c \exp \left(X_{i} \xi_{2}+\kappa_{2} g_{i}\right)\right\},
\end{aligned}
$$

where $\kappa_{1}$ and $\kappa_{2}$ are scale factors. The model (32) now represents (26). Given (32)-(33), and assuming conditional independence between $T$ and $C$, (28) can easily be computed as in (30):

$$
\begin{aligned}
f\left(c_{i}, T_{i} \geq C_{i} \mid g_{i}\right)= & \lambda_{2} \exp \left(X_{i} \xi_{2}+\kappa_{2} g_{i}\right) \exp \left\{-\lambda_{2} c \exp \left(X_{i} \xi_{2}+\kappa_{2} g_{i}\right)\right\} \\
& \times \exp \left\{-\lambda_{1} \exp \left(X_{i} \xi_{1}+\kappa_{1} g_{i}\right) c\right\} .
\end{aligned}
$$

Finally, we formulate a model for the dropout indicator. Let 0 indicate that a measurement has been observed at that occasion, and 1 that the patient has dropped out at that occasion. Then, $\boldsymbol{R}_{i}$ consists of zeros, followed by a single 1 . For someone completing the scheduled visits, the entire sequence would consist of $0 \mathrm{~s}$. We assume a logistic regression model of the form (Creemers et al., 2011):

$$
\operatorname{logit}\left\{P\left(R_{i j}=1 \mid R_{i j-1}=0\right)\right\}=\zeta_{0}+\kappa_{3} g_{i}+\zeta_{1} t_{i j}+\zeta_{2} X_{i}+\zeta_{3} X_{i} t_{i j},
$$

where $\kappa_{3}$ is a scale parameter. Model (35) represents (24).

This now completes the specification of the identifiable components of the model. We refer to this as model (A). The model was fitted using the NLMIXED procedure in SAS, version 9.3. Both prothrombin and time were rescaled to the unit interval, for numerical stability and without statistical or substantive consequences. The results are provided in Table 1 in the column "(A) MAR." It is notable that the three scale parameters are highly statistically significant. Given that the estimates are negative, a higher patient-specific prothrombin profile then corresponds to a reduced hazard of death and of censoring, and reduced odds of drop-out.

The nonidentifiable components still remain. Similar to pattern-mixture models, these can be identified only by unverifiable identifying restrictions. Consider the longitudinal process, $\boldsymbol{Y}_{i}$, partitioned into the observed and missing components $\boldsymbol{Y}_{i}^{o}$ and $\boldsymbol{Y}_{i}^{m}$, respectively. Assuming a linear-mixed model framework such as described earlier, and invoking properties of the multivariate normal distribution, it is easy to derive the predictive distribution of the missing outcomes, given the observed ones. In particular, such a derivation can be done assuming either MAR or MNAR. For example, under MAR, the conditional mean $E\left(Y_{i j}^{m} \mid \boldsymbol{y}_{i}{ }^{o}\right)$ needs to be calculated marginalized over $g_{i}$, whereas under MNAR, one can consider the mean $E\left(Y_{i j}^{m} \mid \boldsymbol{y}_{i}{ }^{o}, g_{i}\right)$, conditional on the random effect. The second choice deviates from what is prescribed by (22) and hence is of MNAR type. Because our longitudinal model is of a random-intercepts type, and hence the corresponding marginal model, marginalized 
Table 1 Liver Cirrhosis Data. Parameter estimates (standard errors) for an MAR analysis, a conventional joint model for the longitudinal and time-to-event outcome, and one where also the censoring time is modeled (extended JM).

\begin{tabular}{|c|c|c|c|c|}
\hline Effect & Parameter & $\begin{array}{l}\text { (A) } \\
\text { MAR }\end{array}$ & $\begin{array}{l}\text { (B) } \\
\text { Conventional JM }\end{array}$ & $\begin{array}{l}\text { (C) } \\
\text { Extended JM }\end{array}$ \\
\hline \multicolumn{5}{|c|}{ Longitudinal process (prothrombin) } \\
\hline Intercept & $\beta_{0}$ & $0.4278(0.0082)$ & $0.4326(0.0080)$ & $0.4330(0.0079)$ \\
\hline Time & $\beta_{1}$ & $0.0635(0.0178)$ & $0.0967(0.0175)$ & $0.1027(0.0177)$ \\
\hline Treatment & $\beta_{2}$ & $-0.0390(0.0117)$ & $-0.0409(0.0114)$ & $-0.0405(0.0113)$ \\
\hline Treatment-by-time & $\beta_{3}$ & $0.0320(0.0258)$ & $0.0315(0.0257)$ & $0.0370(0.0258)$ \\
\hline Measurement error & $\sigma^{2}$ & $0.0113(0.0003)$ & $0.0111(0.0003)$ & $0.0111(0.0003)$ \\
\hline \multicolumn{5}{|l|}{ Survival time } \\
\hline Treatment & $\xi_{1}$ & $0.0142(0.1502)$ & $0.0493(0.1378)$ & $0.0324(0.1214)$ \\
\hline Scale & $\lambda_{1}$ & $2.3331(0.2515)$ & $1.9817(0.2161)$ & $4.2064(0.4192)$ \\
\hline Shape & $\rho_{1}$ & & $0.9076(0.0443)$ & $0.9569(0.0455)$ \\
\hline Scale factor & $\kappa_{1}$ & $-8.5000(0.7232)$ & $-6.7345(0.6980)$ & $-2.6694(0.6496)$ \\
\hline \multicolumn{5}{|l|}{ Censoring time } \\
\hline Treatment & $\xi_{2}$ & $-0.1424(0.1550)$ & & $-0.1572(0.1477)$ \\
\hline Scale & $\lambda_{2}$ & $4.2095(0.4983)$ & & $3.6254(0.4291)$ \\
\hline Shape & $\rho_{2}^{2}$ & & & $1.0450(0.0638)$ \\
\hline Scale factor & $\kappa_{2}$ & $-4.8568(0.9870)$ & & $-2.8537(0.8941)$ \\
\hline \multicolumn{5}{|l|}{ Dropout process } \\
\hline Intercept & $\zeta_{0}$ & $-2.1166(0.1161)$ & & \\
\hline Time & $\zeta_{1}$ & $2.6940(0.3535)$ & & \\
\hline Treatment & $\zeta_{2}$ & $-0.2015(0.1656)$ & & \\
\hline Treatment-by-time & $\zeta_{3}$ & $0.5807(0.4862)$ & & \\
\hline Scale factor & $\kappa_{2}$ & $-4.7932(0.6159)$ & & \\
\hline \multicolumn{5}{|l|}{ Shared effect } \\
\hline Variance of random effects & $\sigma_{g}^{2}$ & $0.0129(0.0010)$ & $0.0123(0.0010)$ & $0.0119(0.0009)$ \\
\hline
\end{tabular}

over the random effect $g_{i}$ has a compound-symmetry structure, Creemers et al. (2010) showed that the predictive distributions are:

$$
\begin{aligned}
& \boldsymbol{Y}_{i}^{m} \mid \boldsymbol{y}_{i}^{o}, g_{i} \sim N\left(\beta_{0}+\beta_{1} v_{i j}+\beta_{2} X_{i}+\beta_{3} X_{i} v_{i j}+g_{i}, \sigma^{2} I_{i}\right), \\
& \boldsymbol{Y}_{i}^{m} \mid \boldsymbol{y}_{i}^{o} \sim N\left(\beta_{0}+\beta_{1} v_{i j}+\beta_{2} X_{i}+\beta_{3} X_{i} v_{i j}, \sigma_{g}^{2} J_{i}+\sigma^{2} I_{i}\right),
\end{aligned}
$$

with $I_{i}$ an identity matrix, $J_{i}$ a matrix of $1 \mathrm{~s}$ with dimensions equal to the number of missing measurements for subject $i$, and the mean function resulting from (31). Recall that the fit to the observed data will not be affected by the choice between both, as the difference between both is entirely in terms of the so-called predictive distribution, that is the distribution of what is unobserved, given what is observed.

Based on (36)-(37), we illustrate the MAR versus MNAR choices graphically, for six censored patients, three from each treatment arm. Their estimated prothrombin mean profiles are extended beyond the point of censoring, under the MAR and MNAR assumptions. In Figure 1, the estimated extended prothrombin mean profiles for these six patients, both under the MNAR and MAR models, are presented. The MNAR model predicts different trajectories, while the predicted trajectories under 


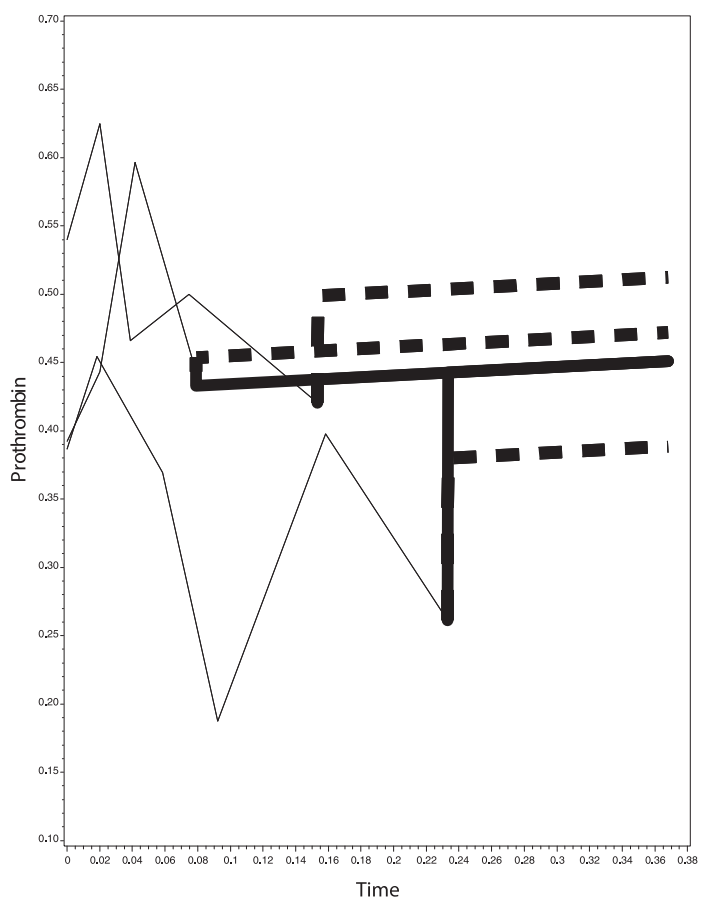

(a) Placebo arm

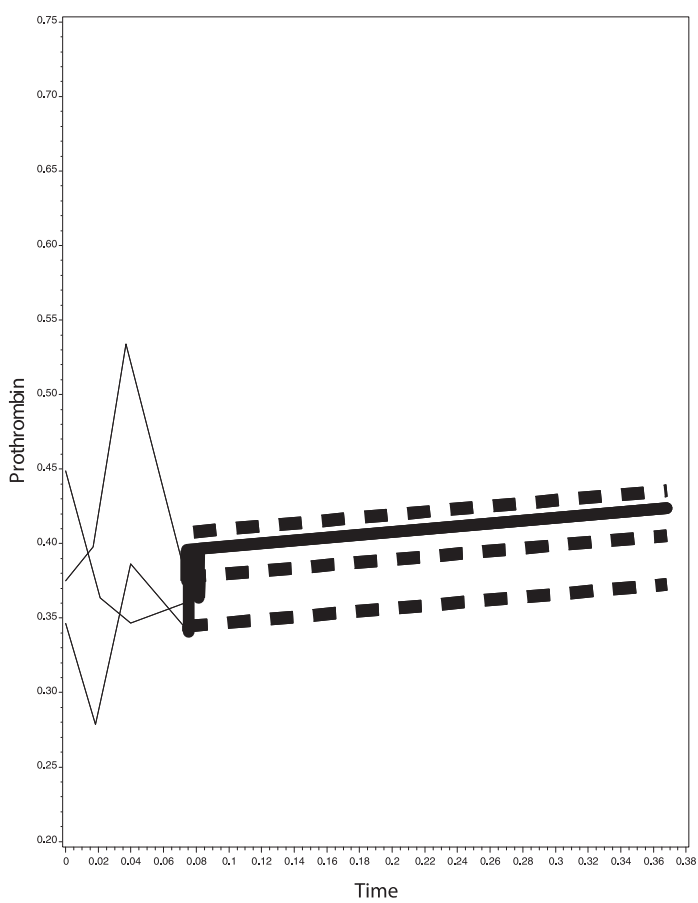

(b) Treatment arm

Figure 1 Extrapolation of prothrombin profiles based on the MNAR (bold dashed lines) and its corresponding MAR model (bold solid lines). Each line brings together the patient's observed measurements and the predicted postcensoring profile.

MAR reduce to a common profile. These observations originate from the fact that the subject-specific effect $g_{i}$ (random effects) come into play under the MNAR models, while the same is not the case for MAR.

The MNAR model considered here is not the only option. One could, for example, also consider more conventional joint models. Two such models have been added to Table 1. In both, a Weibull model is formulated for the event time:

$$
f_{i}\left(t \mid g_{i}\right)=\lambda_{1} \rho_{1} t^{\rho_{1}-1} \exp \left(X_{i} \xi_{1}+\kappa_{1} g_{i}\right) \exp \left\{-\lambda_{1} t^{\rho_{1}} \exp \left(X_{i} \xi_{1}+\kappa_{1} g_{i}\right)\right\} .
$$

In the first conventional model, denoted by (B), censoring is assumed noninformative, with contribution to the likelihood derived from:

$$
S_{i}\left(c \mid g_{i}\right)=\exp \left\{-\lambda_{1} t^{\rho_{1}} \exp \left(X_{i} \xi_{1}+\kappa_{1} g_{i}\right)\right\} .
$$

In the extended version of this model, referred to as $(\mathrm{C})$, the censoring time is described via a Weibull model as well:

$$
f_{i}\left(c \mid g_{i}\right)=\lambda_{2} \rho_{2} c^{\rho_{2}-1} \exp \left(X_{i} \xi_{2}+\kappa_{2} g_{i}\right) \exp \left\{-\lambda_{2} t^{\rho_{2}} \exp \left(X_{i} \xi_{2}+\kappa_{2} g_{i}\right)\right\} .
$$

Models (B) and (C) are shared-parameter models because $g_{i}$ is common to the event-time and longitudinal models, the latter of which still formulated by (31). Note that both models are automatically of an MNAR type, because no measures have been taken to restrict the impact of the random effect $g_{i}$. 
In all three models, inferences of, for example, the treatment effect and treatment-by-time interactions is qualitatively the same. This strengthens the evidence that the findings are stable and trustworthy. The advantage of model (A) is that it allows to construct predictions under the MAR assumption, and examine how these change for specific choices of MNAR predictions.

\section{Concluding remarks}

In this paper, we have used the strong connection between the longitudinal, time-to-event, and missing data settings, to build an extended shared random effects joint model, similar in spirit to that of Creemers et al. (2011) in the context of longitudinal data subject to missing observations, but now transposed to the current more complex setting. In doing so, we have taken a slightly different perspective on joint models than is prevalent in the literature.

Within this extended framework, we have provided a characterization of MAR, consistent to the one in the missing data setting. While the framework has been built conceptually, we have illustrated the ideas through an analysis of data from a study on liver cirrhosis. We have also illustrated the implications of MAR and MNAR.

It is still possible, within the elaborate random effects structure, to formulate additional alternative models, all making different assumptions. The stability of inferences across such models can then be considered. This provides a rich avenue for sensitivity analyses. For example, we were able to contrast our MAR model (A), with its MNAR counterpart (graphically), as well as with more conventional joint models (B) and (C). The fact that inferences about scientific parameters remain roughly unaltered, strengthens confidence in the findings.

Finally, the method proposed in this paper comes at a computational cost. Even though it is possible to use, without too much difficulty, existing statistical software, multi-dimensional random effects structures will make the method more complicated. This is not particular to the proposed model but rather to any mixed model that is at least as complex as a generalized linear-mixed model with increasing dimension of the random-effects vector. Various authors have considered ways to simplify the model fitting. This includes, for example, pseudo-likelihood or composite likelihood, an account of which is given in Molenberghs and Verbeke (2005) and Molenberghs et al. (2011). Further treatment of this topic is outside the scope of this paper.

SAS code for models (A) and (C) can be found in the Supplementary Materials.

Acknowledgments We gratefully acknowledge support from IAP research Network P7/06 of the Belgian Government (Belgian Science Policy). We also thank the referees for their helpful comments.

\section{Conflict of interest}

The authors have declared no conflict of interest.

\section{References}

Andersen, P., Borgan, O., Gill, R. and Keiding, N. (1993). Statistical Models Based on Counting Processes. Springer, New York, NY.

Creemers, A., Hens, N., Aerts, M., Molenberghs, G., Verbeke, G. and Kenward, M. G. (2011). Generalized shared-parameter models and missingness at random. Statistical Modeling 11, 279-311.

Creemers, A., Hens, N., Aerts, M., Molenberghs, G., Verbeke, G. and Kenward, M. G. (2010). A sensitivity analysis for shared-parameter models for incomplete longitudinal outcomes. Biometrical Journal 52, 111-125.

DeGruttola, V. and Tu, X. M. (1994). Modeling progression of CD4 lymphocyte count and its relationship to survival time. Biometrics 50, 1003-1014.

Duchateau, L. and Janssen, P. (2008). The Frailty Model. Springer, New York, NY. 
Faucett, C. L. and Thomas, D. C. (1996). Simultaneously modeling censored survival data and repeatedly measured covariates: a Gibbs sampling approach. Statistics in Medicine 15, 1663-1685.

Henderson, R., Diggle, P. and Dobson, A. (2000). Joint modeling of longitudinal measurements and event time data. Biostatistics 1, 465-480.

Hogan, J. W. and Laird, N. M. (1997). Mixture models for the joint distribution of repeated measures and event times. Statistics in Medicine 16, 239-258.

Hogan, J. W. and Laird, N. M. (1998). Increasing efficiency from censored survival data by using random effects to model longitudinal covariates. Statistical Methods in Medical Research 7, 28-48.

Klein, J. P. and Moeschberger, M. L. (1997). Survival Analysis: Techniques for Censored and Truncated Data. Springer, New York, NY.

Lavalley, M. P. and DeGruttola, V. (1996). Models for empirical Bayes estimators of longitudinal CD4 counts. Statistics in Medicine 15, 2289-2305.

Law, N. J., Taylor, J. M. G. and Sandler, H. (2002). The joint modeling of a longitudinal disease progression marker and the failure time process in the presence of cure. Biostatistics 3, 547-563.

Little, R. J. A. and Rubin, D. B. (2002). Statistical Analysis with Missing Data. John Wiley \& Sons, New York, NY.

Yu, M., Taylor, J. M. G. and Sandler, H. M. (2008). Individual prediction in prostate cancer studies using a joint longitudinal survival cure model. Journal of the American Statistical Association 103, 178-187.

Molenberghs, G., Beunckens, C., Sotto, C. and Kenward, M. G. (2008). Every missing not at random model has got a missing at random counterpart with equal fit. Journal of the Royal Statistical Society, Series B 70, 371-388

Molenberghs, G. and Verbeke, G. (2005). Models for Discrete Longitudinal Data. Springer, New York, NY.

Molenberghs, G. and Kenward, M. G. (2007). Missing Data in Clinical Studies. John Wiley \& Sons, New York, NY.

Molenberghs, G., Njeru, N. E., Kenward, M. G. and Verbeke, G. (2012). Enriched-data problems and essential non-identifiability. International Journal of Statistics in Medical Research 1, 16-44.

Molenberghs, G., Michiels, B., Kenward, M. G. and Diggle, P. J. (1998). Monotone missing data and patternmixture models. Statistica Neerlandica 52, 153-161.

Molenberghs, G. and Verbeke, G. (2005). Models for Discrete Longitudinal Data. Springer, New York, NY.

Molenberghs, G. and Verbeke, G. (2004) Meaningful statistical model formulations. Statistica Sinica 14, 989-1020.

Molenberghs, G., Verbeke, G. and Iddi, S. (2011). Pseudo-likelihood methodology for partitioned large and complex samples. Statistics \& Probability Letters 81, 892-901.

Njagi, E. N., Molenberghs, G., Rizopoulus, D., Verbeke, G., Kenward, M. G., Dendale, P. and Willekens, K. (2013). A flexible joint-modeling framework for longitudinal and time-to-Event data with overdispersion. Statistical Methods in Medical Research. To appear.

Pawitan, Y. and Self, S. (1993). Modeling disease marker processes in AIDS. Journal of the American Statistical Association 88, 719-726.

Rizopoulos, D. (2012). Joint Models for Longitudinal and Time-to-Event Data. Chapman and Hall/CRC, Boca Raton, FL.

Rizopoulos, D., Verbeke, G. and Lesaffre, E. (2009). Fully exponential Laplace approximations for the joint modelling of survival and longitudinal data. Journal of the Royal Statistical Society, Series B 71, 637-654.

Rizopoulos D. (2011). Dynamic predictions and prospective accuracy in joint models for longitudinal and timeto-event data. Biometrics 67, 819-829.

Rubin, D. B. (1976). Inference and missing data. Biometrika 63, 581-592.

Taylor, J. M. G. Cumberland, W. G. and Sy, J. P. (1994). A stochastic model for analysis of longitudinal AIDS data. Journal of the American Statistical Association 89, 727-736.

Tsiatis, A. A. (1975). A nonidentifiability aspect of the problem of competing risks. Proceedings of the National Academy of Sciences 72, 20-22.

Tsiatis, A. and Davidian, M. (2004). Joint modeling of longitudinal and time-to-event data: an overview. Statistica Sinica 14, 809-834.

Tsiatis, A. A., DeGruttola, V. and Wulfsohn, M. S. (1995). Modeling the relationship of survival to longitudinal data measured with error. Applications to survival and CD4 counts in patients with AIDS. Journal of the American Statistical Association 90, 27-37.

Verbeke, G. and Molenberghs, G. (2000). Linear Mixed Models for Longitudinal Data. Springer, New York, NY. 
Verbeke, G. and Molenberghs, G. (2011). Arbitrariness of models for augmented and coarse data, with emphasis on incomplete-data and random-effects models. Statistical Modelling 10, 391-419.

Verbeke, G., Molenberghs, G. and Rizopoulos, D. (2010). Random effects models for longitudinal data. Longitudinal Research with Latent Variables. K. van Montfort, H. Oud, and Al Satorra (Eds.). Springer, New York, NY, pp. 37-96.

$\mathrm{Xu}$, J. and Zeger, S. L. (2001). Joint analysis of longitudinal data comprising repeated measures and times to events. Applied Statistics 50, 375-387.

Yu, M., Law, N. J., Taylor, J. M. G. and Sandler, H.M. (2004). Joint longitudinal-survival-cure models and their application to prostate cancer. Statistica Sinica 14, 835-862. 